\title{
White matter pathways for prosodic structure building: A case study
}

Daniela Sammler $^{\mathrm{a}, \mathrm{b}}$, Katrin Cunitz ${ }^{\mathrm{b}, \mathrm{c}}$, Sarah M. E. Gierhan ${ }^{\mathrm{b}, \mathrm{d}}$, Alfred Anwander ${ }^{\mathrm{b}}$, Jens Adermann ${ }^{\mathrm{e}}$, Jürgen Meixensberger ${ }^{\mathrm{e}}$, Angela D. Friederici ${ }^{\mathrm{b}, \mathrm{d}}$

a Otto Hahn Group "Neural Bases of Intonation in Speech and Music", Max Planck Institute for Human Cognitive and Brain Sciences, Stephanstraße 1a, 04103 Leipzig, Germany

${ }^{b}$ Department of Neuropsychology, Max Planck Institute for Human Cognitive and Brain Sciences, Stephanstraße 1a, 04103 Leipzig, Germany

${ }^{c}$ Department of Child and Adolescent Psychiatry and Psychotherapy, University Hospital Ulm, Steinhövelstraße 5, 89075 Ulm, Germany

${ }^{d}$ Berlin School of Mind and Brain, Humboldt University Berlin, Unter den Linden 6, 10099 Berlin, Germany

e University Hospital Leipzig, Clinic and Policlinic for Neurosurgery, Liebigstraße 20, 04103 Leipzig, Germany

Running title: Fiber tracts for prosodic structure building

Corresponding author:

Dr Daniela Sammler

Max Planck Institute for Human Cognitive and Brain Sciences

Stephanstraße 1a, 04103 Leipzig, Germany

Email: sammler@cbs.mpg.de

Phone: +49 34199402679

Fax: +4934199402204 


\begin{abstract}
The relevance of left dorsal and ventral fiber pathways for syntactic and semantic comprehension is well established, while pathways for prosody are little explored. The present study examined linguistic prosodic structure building in a patient whose right arcuate/superior longitudinal fascicles and posterior corpus callosum were transiently compromised by a vasogenic peritumoral edema. Compared to ten matched healthy controls, the patient's ability to detect irregular prosodic structure significantly improved between pre- and post-surgical assessment. This recovery was accompanied by an increase in average fractional anisotropy (FA) in right dorsal and posterior transcallosal fiber tracts. Neither general cognitive abilities nor (non-prosodic) syntactic comprehension nor FA in right ventral and left dorsal fiber tracts showed a similar pre-post increase. Together, these findings suggest a contribution of right dorsal and inter-hemispheric pathways to prosody perception, including the right-dorsal tracking and structuring of prosodic pitch contours that is transcallosally informed by concurrent syntactic information.
\end{abstract}

Keywords: language; prosody; voice; pitch; DTI; auditory pathways; arcuate fascicle; dorsal stream; corpus callosum 


\section{Introduction}

White-matter fiber bundles connecting left fronto-temporal (and parietal) 'language areas' have become a centerpiece of modern language models (Friederici, 2011) and their divide into functionally specialized dorsal and ventral routes is largely undisputed (Hickok \& Poeppel, 2007; Rauschecker \& Scott, 2009). However, the established roles of left dorsal and ventral fiber tracts in speech production and semantic comprehension (Fridriksson et al., 2018; Kümmerer et al., 2013; Saur et al., 2008), as well as syntactic parsing (Friederici, 2012; Griffiths, Marslen-Wilson, Stamatakis, \& Tyler, 2013; Wilson et al., 2011) ignore one important component of spoken language: Speech prosody, the rhythmic-melodic variations in speech that serve linguistic functions ${ }^{1}$ (Cutler, Dahan, \& Van Donselaar, 1997). The notable involvement of right-hemispheric fronto-temporal brain areas in linguistic prosodic processing (for reviews, see Baum \& Pell, 1999; Belyk \& Brown, 2014; Paulmann, 2016; Witteman, van ljzendoorn, van de Velde, van Heuven, \& Schiller, 2011) calls for reflection upon the contribution of right-hemispheric (Sammler, Grosbras, Anwander, Bestelmeyer, \& Belin, 2015) as well as inter-hemispheric pathways to natural language comprehension (Friederici \& Alter, 2004). Here, we present a case study that lends evidence for the functional necessity of right dorsal and transcallosal pathways in linguistic prosodic structure building.

Speakers naturally vary prosodic features such as intonation and rhythm in their utterances to package information into meaningful units and to accentuate thematically relevant words (Cole, 2015; Cutler et al., 1997; Wagner \& Watson, 2010). Indeed, we do well in relying on prosodic cues in ambiguous sentences like "Wave at the girl with the hat." to understand at which girl we should wave and in which way (Lehiste, 1973; Snedeker \& Trueswell, 2003). This example is one of many to illustrate how the prosodic structure of an utterance, i.e., its organization into smaller phonological or intonational phrases (Selkirk, 1996), can assist language comprehension: The systematic alignment of prosodic phrase boundaries with syntactic and semantic structure (Cooper \& Paccia-Cooper, 1980; Selkirk, 1984; Watson \& Gibson, 2004) allows listeners to use prosody in their syntactic and semantic interpretation, and vice versa (Buxó-Lugo \& Watson, 2016; Cole, Mo, \& Baek, 2010). Changes in pitch contour, pre-boundary lengthening and pauses are amongst the most important acoustic cues that signal prosodic boundaries (Ladd, 2008; Pierrehumbert \& Hirshberg, 1990) and constrain parsing possibilities.

\footnotetext{
${ }^{1}$ Prosody also conveys paralinguistic information about speakers' emotions, attitudes, and intentions (Hellbernd \& Sammler, 2016; Scherer, 1986) which will not be addressed in the present study.
} 
The tracking of these cues has often been associated with the right hemisphere, in line with cuedependent models of auditory speech perception (Friederici \& Alter, 2004; McGettigan \& Scott, 2012; Poeppel, 2003; Zatorre, Belin, \& Penhune, 2002). These models argue for a relative processing benefit of right auditory cortices for pitch and spectral information (Jamison, Watkins, Bishop, \& Matthews, 2006; Johnsrude, Penhune, \& Zatorre, 2000; Obleser, Eisner, \& Kotz, 2008; Schönwiesner, Rübsamen, \& von Cramon, 2005; Zatorre et al., 2002) that unfolds over extended timescales (Giraud et al., 2007; Poeppel, 2003). As a consequence, the right hemisphere may optimally track suprasegmental prosodic features and complement left-hemispheric syntactic and semantic processes, as proposed in the Dynamic Dual Pathway Model of Friederici and Alter (2004). In keeping with this hemispheric division of labor, fMRI and dichotic listening studies reported predominant right fronto-temporal activations (Kyong et al., 2014; Meyer, Alter, Friederici, Lohmann, \& von Cramon, 2002; Meyer, Steinhauer, Alter, Friederici, \& von Cramon, 2004; Plante, Creusere, \& Sabin, 2002) and a left ear advantage (i.e., right hemisphere involvement; Blumstein \& Cooper, 1974; Shipley-Brown, Dingwall, Berlin, Yeni-Komshian, \& GordonSalant, 1988) when listening to filtered or degraded speech with high demands on prosodic processing. Likewise, explicit attention to prosodic pitch contours in statements and questions (compared to processing of phonemes and lexical meaning) induced right-lateralized activity in fronto-temporal regions (Kreitewolf, Friederici, \& von Kriegstein, 2014; Sammler et al., 2015). The right-lateralization of prosody is less clear-cut in studies with natural language material (e.g., Perkins, Baran, \& Gandour, 1996; Tang, Hamilton, \& Chang, 2017), prosody production (Kellmeyer et al., 2013; Peschke, Ziegler, Eisenberger, \& Baumgaertner, 2012), and tasks that go beyond the processing of low-level acousticprosodic cues such as pitch contour (for reviews showing bilateral involvement, see Baum \& Pell, 1999; Belyk \& Brown, 2014; Paulmann, 2016; Witteman et al., 2011). This indicates the inevitable interaction of prosodic information with concurrent syntactic (den Ouden, Dickey, Anderson, \& Christianson, 2016) or lexical-semantic processes (Domahs, Klein, Huber, \& Domahs, 2013; Gandour et al., 2004; van Lancker, 1980) that are hard to separate during natural language comprehension.

The present study focuses on sentence-level prosodic structure building, i.e., the gradual emergence of a (hierarchical) representation of prosodic constituency that aligns with syntactic structure. As outlined above and implied by previous psycholinguistic research, the prosodic parser most likely draws both on acoustic markers for prosodic boundaries (Ladd, 2008; Pierrehumbert \& Hirshberg, 1990; Snedeker \& Trueswell, 2003) as well as concurrent syntactic structure (Buxó-Lugo \& Watson, 2016; Cole et al., 2010) to build prosodic representations. At the neural level, this implies involvement of both right-hemispheric 
fronto-temporal networks that track relevant prosodic features over time as well as inter-hemispheric exchange to map syntactic and prosodic structure onto each other (Friederici \& Alter, 2004).

This assumption naturally raises the question how information is transferred between relevant brain areas. Syntactic structure building in the left hemisphere is known to involve ventral fronto-temporal connections via the inferior fronto-occipital (IFOF) and uncinate fascicles (UF) for simple syntactic parsing, while dorsal connections via the arcuate and superior longitudinal fascicles (AF/SLF) support parsing of complex syntactic structures (Friederici, 2012; Griffiths et al., 2013) (for reviews, see Friederici, 2011; Gierhan, 2013b). Correspondingly, damage to left dorsal fiber tracts (Meyer, Cunitz, Obleser, \& Friederici, 2014; Wilson et al., 2011) or their developmental immaturity (Skeide, Brauer, \& Friederici, 2016) coincide with reduced comprehension of syntactically complex sentences.

Recently, we demonstrated a similar multi-pathway architecture in the right hemisphere for the perception of prosodic pitch contours in statements and questions (Sammler et al., 2015). This finding was remarkable because the relevance of right-hemispheric and particularly right dorsal tracts in speech and language has been questioned until very recently (Hickok, 2012). Indeed, direct right dorsal frontotemporal connections were often found to be anatomically weaker than their left-hemispheric counterparts (Fernández-Miranda et al., 2015; Glasser \& Rilling, 2008; Parker et al., 2005; Powell et al., 2006; Thiebaut de Schotten, Ffytche, et al., 2011) and have been studied nearly exclusively in the context of atypical language lateralization (Duffau, Leroy, \& Gatignol, 2008; Vassal, Le Bars, MoritzGasser, Menjot, \& Duffau, 2010), e.g., during aphasia rehabilitation (Forkel et al., 2014; Schlaug, Marchina, \& Norton, 2009). What has remained unexplored so far is the potential contribution of right dorsal tracts to the processing of suprasegmental prosodic information in speech. Our data on statement and question discrimination lend initial evidence for that, albeit only for single words (Sammler et al., 2015; for converging evidence in emotional prosody perception, see Frühholz, Gschwind, \& Grandjean, 2015; Glasser \& Rilling, 2008). It seems plausible, though, that the capacity of (right) AF/SLF and temporal-premotor loops to constantly monitor sound and pitch (Guenther \& Vladusich, 2012; Houde \& Chang, 2015; Zarate, 2013) may benefit the acoustic detection of prosodic boundaries in sentences. A yet bolder proposal that awaits testing is the potential involvement of right dorsal fronto-temporal tracts in more advanced prosodic structuring, beyond basic pitch tracking (Bornkessel-Schlesewsky \& Schlesewsky, 2013).

The interaction between the lateralized syntax and prosody streams requires a dynamic exchange between the two hemispheres (Friederici \& Alter, 2004; Steinmann \& Mulert, 2012). Several studies 
suggest that syntax-prosody alignment hinges particularly on the cross-talk between the temporal lobes via commissural fibers that cross through the posterior third of the corpus callosum (CC; Friederici, von Cramon, \& Kotz, 2007; Sammler, Kotz, Eckstein, Ott, \& Friederici, 2010; for the anatomy of CC, see Hofer \& Frahm, 2006; Huang et al., 2005). Accordingly, patients with permanent lesions in the posterior CC no longer processed prosodic (or syntactic) irregularities that were only detectable if the syntactic (or prosodic) context was taken into account (Friederici et al., 2007; Sammler et al., 2010). The present study extends these findings to a new case with temporary dysfunctions of relevant white matter tracts.

We report the case of a patient in whom right dorsal and transcallosal connectivity were transiently compromised due to a vasogenic peritumoral edema, allowing assessment of potential prosodic deficits and their recovery in the same individual. Vasogenic edemas are extracellular edemas; other than cytotoxic edemas they infiltrate white matter, not cell bodies (Stokum, Gerzanich, \& Simard, 2016), i.e., leave the neurons largely intact if the edema is medically treated to induce its reabsorption. Nevertheless, vasogenic edemas can compromise function in that they compress tissue and disturb information flow along the infiltrated white matter tracts. While the underlying neurophysiological mechanisms are still not fully understood, resulting physical or cognitive deficits are typically alleviated over the course of edema reabsorption (e.g., Bizzi et al., 2012). We capitalized on this phenomenon to probe the involvement of right dorsal and inter-hemispheric tracts in prosody perception in a patient with vasogenic edema infiltrating right $\mathrm{AF} / \mathrm{SLF}$ and the posterior corpus callosum.

One important consideration for our investigation is that vasogenic edemas are seen around brain tumors. Our patient was diagnosed with a benign convexity meningioma (grade I) in the right parietal region. This type of meningioma is a slow-growing tumor on the surface of the brain, i.e., not invading grey matter. In line with a slow growth rate, symptoms typically have an insidious onset such as slowly evolving headache, suggestive of increased intracranial pressure, or a protracted history of partial seizures (Rockhill, Mrugala, \& Chamberlain, 2007). Complete excision of the meningioma is often curative. Slow growth allows for functional compensation; among intracranial tumors, meningiomas are the ones with the highest incidental discovery rate, and can remain neurologically and cognitively asymptomatic, especially if located in the right hemisphere (Nishizaki, Ozaki, Kwak, \& Ito, 1999). Overall, the long-term course of meningioma with potential for compensation make it likely that post-surgical reversal of pre-surgical deficits in our patient are due to edema absorption, i.e., relief from edemainduced compression and recovery of white matter tracts, rather than removal of the tumor. 
The present study combined pre- and post-surgical diffusion-weighted neuroimaging with behavioral assessment of linguistic prosody perception in a male patient with a right parietal edema infiltrating $\mathrm{AF} / \mathrm{SLF}$ and the posterior corpus callosum. Specifically, we assessed the patient's ability to detect prosodic phrase boundaries that mismatched syntactic phrase structure (Eckstein \& Friederici, 2006), requiring both right-hemispheric prosodic contour processing as well as syntax-prosody alignment across both hemispheres. To rule out general left-hemispheric syntax processing deficits, a non-prosodic control task was designed that tested comprehension of sentences with canonical and non-canonical syntactic structure (Gierhan, 2013a). The patient's performance was compared with performance of ten matched healthy controls $(\mathrm{HC})$ that were tested twice at the same interval as the patient, to control for learning effects. General cognitive functioning was assessed with a standard neuropsychological test battery. If right dorsal and/or transcallosal pathways are essential for prosodic structure building and vasogenic edemas compromise white matter function, the patient's prosody perception should (i) be deficient in the pre-surgical but normal in the post-surgical session compared to HC, should (ii) increase more strongly between pre- and postsurgical session than in HC (who could show learning effects), and should (iii) increase more strongly than in the non-prosodic syntax task and neuropsychological tests.

\section{Materials and methods}

\subsection{Participants}

Our patient (male, 43 years, right-handed) presented for assessment of two generalized epileptic seizures at the neurosurgical department of the University Hospital Leipzig. A computer tomography (CT) of the brain revealed a vasogenic edema $\left(37393 \mathrm{~mm}^{3}\right)$ surrounding a benign convexity meningioma $\left(25519 \mathrm{~mm}^{3}\right)$ in the right parietal lobe (Figure 1A; see also Supplementary Figure 1). The edema was immediately admitted to treatment with glucocorticoids/dexamethasone (Meixensberger \& Jaeger, 2005; $48 \mathrm{mg}$ intravenous on the day of diagnosis, then $3 \times 8 \mathrm{mg}$ daily up until surgery). Such a treatment leads to the continuous absorption of the edema within a period of 2-3 weeks, usually associated with a considerable functional recovery that illustrates that the edema causes transient deficits beyond those caused by the tumor. Twenty-one days after diagnosis, the tumor was microsurgically resected. We tested the patient on day 2 and acquired MR images on day 3 after beginning of medication, i.e., when the edema still compromised the dorsal fiber tracts. The second acquisition was 110 days after neurosurgical resection of the tumor and full absorption of the edema (i.e., 134 days after the first session). Ten healthy male control participants (HC) matched in age (mean $\pm S E M: 42 \pm 0.6$ years), years 
of school education (10.2 \pm 0.2 years), and handedness were tested and re-tested with a mean interval of $145 \pm 12$ days between sessions to account for potential learning effects in a test-retest setup. Neither the patient, nor the controls were musicians; none of the controls reported hearing deficits as verified with a MAICO MA 33 audiometer (MAICO Diagnostics GmbH, Berlin, Germany). The patient displayed slightly lower hearing on the left than the right ear $(125 \mathrm{~Hz}$ : left 17.5 / right $32.5 \mathrm{~dB} \mathrm{HL;} 250$ Hz: 20.0 / 37.5 dB HL; 1000 Hz: 25.0 / 45.0 dB HL; 4000 Hz: 17.5 / 55 dB HL). This was accounted for by adjusting the volume of the experimental stimuli to well audible level. All participants gave written informed consent. The study was approved by the ethics committee of the University of Leipzig (017-10180112009).

\subsection{MRI data acquisition}

Anatomical and diffusion MRI data were obtained pre- and post-surgically in the patient and once for seven of the HC. Three HC were not scanned due to MR incompatibility. Data were acquired with a 32channel head coil in a 3 Tesla TIM TRIO scanner (Siemens Healthineers, Erlangen, Germany) at the Max Planck Institute for Human Cognitive and Brain Sciences, Leipzig, Germany. High-resolution $T_{1}$-weighted anatomical datasets $\left(1 \times 1 \times 1 \mathrm{~mm}^{3}\right.$ voxel size) were acquired using a 3D magnetization-prepared rapid gradient echo (MPRAGE) sequence (repetition time TR $=1300 \mathrm{~ms}$, echo time TE $=3.46 \mathrm{~ms}$, 176 sagittal

slices, field of view FOV $=240 \times 256 \mathrm{~mm}^{2}$, flip angle $=10^{\circ}$ ). The diffusion-weighted data sets were acquired with a twice-refocused spin-echo EPI sequence (TR $=12.9 \mathrm{~s}, \mathrm{TE}=100 \mathrm{~ms}, 88$ axial slices without gap, FOV $=220 \times 220 \mathrm{~mm}^{2}$, flip angle $=90^{\circ}$, GRAPPA acceleration factor 2) with a voxel size of $1.72 \times$ $1.72 \times 1.7 \mathrm{~mm}^{3}$. Diffusion-weighting was isotropically distributed along 60 diffusion-encoding gradient directions with a b-value of $1000 \mathrm{~s} / \mathrm{mm}^{2}$. Acquisition of diffusion-weighted images was interspersed with seven images without diffusion-weighting (b0), one at the beginning of the sequence and one after each block of 10 diffusion-weighted images, serving as anatomical reference for offline motion correction. Total duration of both MRI scans was about 25 minutes. 


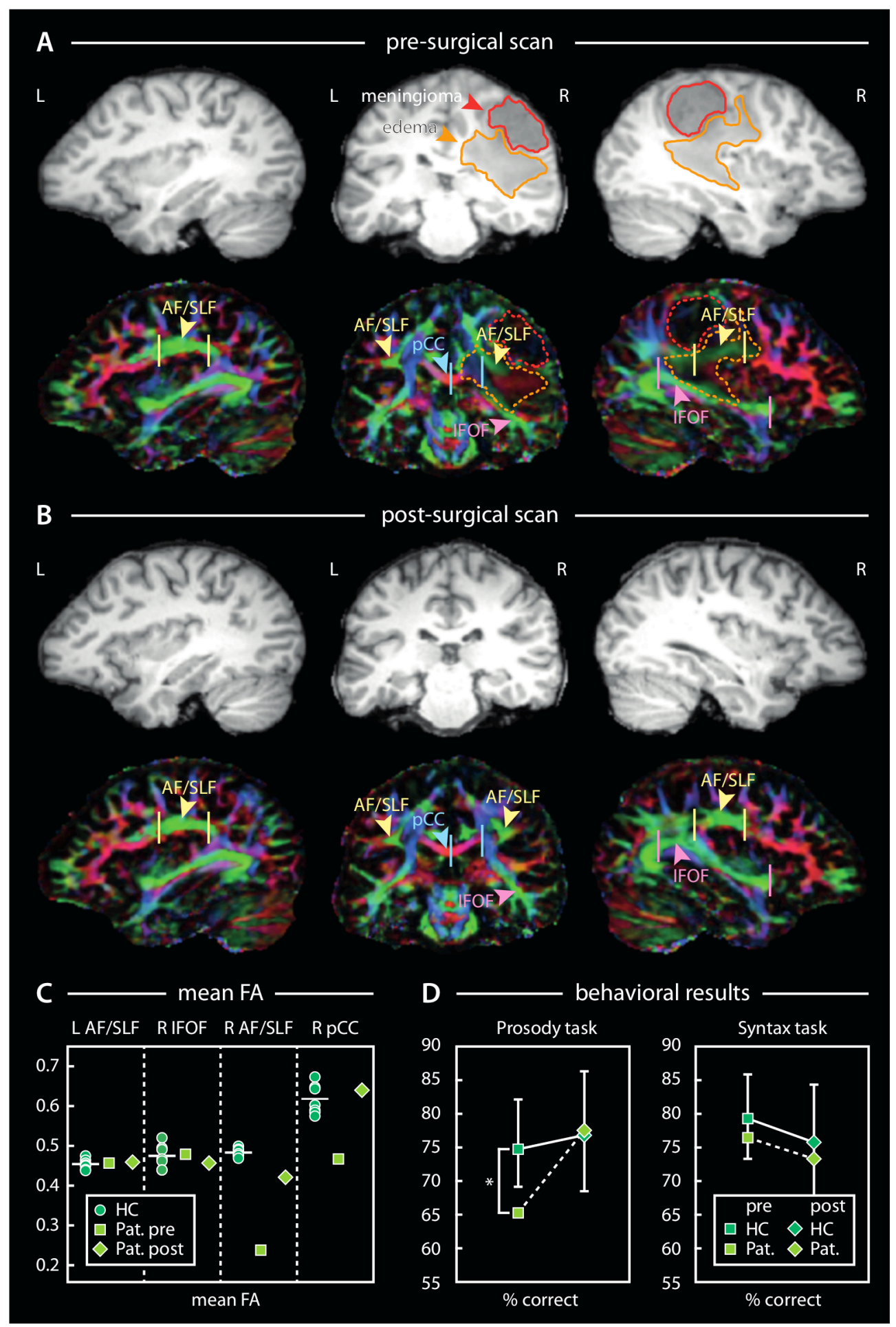

Figure 1. Pre- and post-surgical results. (A) Pre-surgical anatomical (grey) and directionally colored diffusion MR FA-images of the patient. The peritumoral edema (orange) infiltrated right dorsal fronto-temporal/parietal pathways leading to lower fractional anisotropy (FA; dark green) in right compared to left AF/SLF (white arrows). The meningioma (red) did not invade but displaced brain tissue as can be seen in the position of the corpus 
callosum (CC). White bars indicate borders for extraction of mean FA in AF/SLF as depicted in (C). Edema and tumor were manually segmented with itk-SNAP 3.2 (http://www.itksnap.org/pmwiki/pmwiki.php). (B) Postsurgically, the edema was fully reabsorbed and the tumor resected, allowing for a recovery of tissue properties (measured by FA) in right AF/SLF (higher green saturation than in (A)). (C) Pre-surgically low FA in right AF/SLF and posterior CC (pCC) of the patient (green square) approached mean FA values of HC (green circles) in the postsurgical session (green diamond), while left AF/SLF as well as right IFOF showed similar FA values as in HC across both sessions. Horizontal lines indicate mean FA of HC. (D) Behavioral results in the prosody and syntax task. Plots show pre- and post-surgical performance of the patient (dashed line) compared to the $1^{\text {st }}$ and $2^{\text {nd }}$ test session of ten matched healthy controls (solid line). Error bars indicate bootstrapped 95\% confidence intervals.

\subsection{Behavioral assessment}

\subsubsection{Prosody task}

Participants were presented with 96 German sentences spoken by a female native speaker. Half of the sentences contained a prosodic irregularity in that pitch contour signaled sentence closure before all obligatory syntactic elements had occurred (Figure 2). All sentences consisted of a matrix clause including a proper name ('Steffen') and a verb ('sieht'/'sees'), and a subordinate clause including a complementizer ('dass'/'that'), a noun phrase ('der Lehrer'/'the teacher'), a prepositional phrase ('beim Tadel'/'during-the reproval'), and a verb ('schmunzelt'/'smiles'). Sentences were rendered prosodically irregular by inserting a prosodic boundary tone with falling pitch contour on the penultimate noun (i.e., 'Tadel') by means of cross splicing (for details on stimulus preparation and acoustic properties, see Eckstein \& Friederici, 2006). This acoustic manipulation induced a mismatch between syntactic and prosodic structure: Syntax predicted sentence continuation beyond the noun given that at least the obligatory verb ('smiles') was still to follow. As a consequence, the noun's falling prosodic contour violated syntax-driven expectancies of prosodic form (auditory examples are provided as Supplementary Material). Prosodically regular and irregular sentences were presented with equal probability across the experiment in pseudo-random order with no more than three consecutive trials of the same type. Sentences had an average ( \pm SEM) duration of $3368 \pm 19 \mathrm{~ms}$ and were presented binaurally at a comfortable volume via loudspeakers (Eltax HT-1; Eltax, Aulum, Denmark) in a silent room using Presentation 12.2. (Neurobehavioral Systems, Inc., Albany, Canada). Participants were asked to judge the prosodic regularity of the sentences by pressing one of two buttons. The experiment started with ten practice trials (with feedback) to acquaint participants with the task and lasted approximately 15 minutes. 


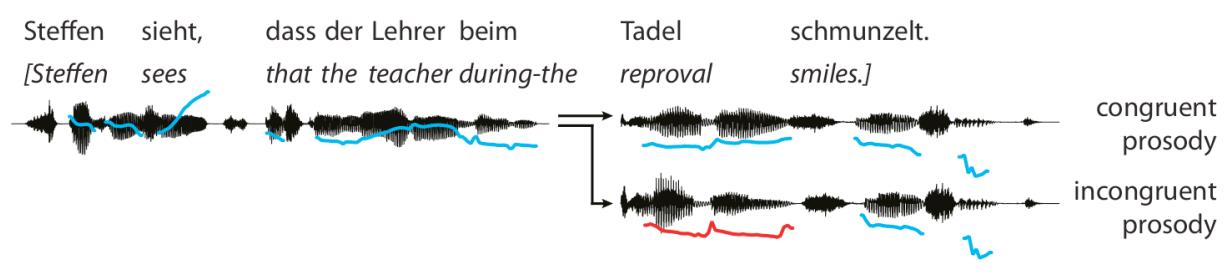

Figure 2. Stimulus examples of the prosody task. The penultimate word of German sentences (here: 'Tadel') was rendered prosodically incongruent (falling instead of rising pitch contour) by means of cross-splicing (Eckstein \& Friederici, 2006).

\subsubsection{Syntax task}

To ensure that putative deficits in detecting prosodic irregularities are not due to a general deficit in processing syntactic information, a non-prosodic sentence comprehension task was adapted from Gierhan (2013a) that is known to involve left (not right) dorsal fiber tracts (Friederici \& Gierhan, 2013). Participants were presented with 90 sentences with canonical and non-canonical syntactic structure spoken by a female native speaker of German. Sentences were composed of a pronoun ('Dann'/'Then'), a verb ('grüßt'/'greets'), an animate subject ('der Soldat'/'the soldier'), and an animate object ('den Major'/'the major'). Half of the sentences presented the subject first, followed by the object (S-O sentences, e.g., 'Dann grüßt $\operatorname{der}_{[S]}$ Soldat $_{[S]} \operatorname{den}_{[O]}$ Major $\left._{[O]}\right]^{\prime} /$ 'Then greets the $e_{[S]}$ soldier $_{[S]}$ the $_{[O]}$ major $_{[O]}$ '). The other half presented the object before the subject (O-S sentences, e.g., 'Dann grüßt den ${ }_{[O]}$ Major $_{[O]}$ $\operatorname{der}_{[s]}$ Soldat $\left._{[S]}\right]^{\prime} /$ 'Then greets the $e_{[0]}$ major $_{[O]}$ the $_{[s]}$ soldier $\left._{[s]}{ }^{\prime}\right)$, which is a legal construction in German. (Note that in both examples, it is the soldier who greets the major.) Sentences were spoken with neutral, non-accentuated prosody (auditory examples are provided as Supplementary Material). After each sentence, participants were asked a comprehension question (spoken by the same female speaker) of the sort 'who did what to whom' to assess their syntactic processing. These questions required either a 'yes' response (50\%), or a 'no' response because it reversed subject and object (25\%; e.g., it was asked whether the major greets the soldier), or introduced a new action or protagonist (25\%; e.g., it was asked whether the soldier blames the major; for examples, see Table 1). Sentences had an average ( \pm SEM) duration of $2619 \pm 12 \mathrm{~ms}$ and were presented in the same way as stimuli in the prosody task. The experiment started with six practice trials (with feedback) and lasted approximately 15 minutes. 
Table 1. Stimulus examples of the syntax task (with literal translations).

(1) Example S-O sentence (subject-first)

Dann grüßt der ${ }_{[\mathrm{S}]}$ Soldat $_{[\mathrm{S}]}$ den $_{[\mathrm{O}]}$ Major $_{[0]}$.

Then greets the $e_{[S]}$ soldier $_{[S]}$ the $_{[0]}$ major $_{[0]}$.

(2) Example O-S sentence (object-first)

Dann grüßt den ${ }_{[\mathrm{O}]}$ Major $_{[0]} \operatorname{der}_{[\mathrm{S}]}$ Soldat $_{[\mathrm{S}]}$.

Then greets the $e_{[0]}$ major $_{[0]}$ the $_{[S]}$ soldier $_{[S]}$.

(3) Examples of questions that require a 'yes' response

Grüßt der $_{[S]}$ Soldat $_{[\mathrm{S}]} \operatorname{den}_{[0]}$ Major $_{[0]}$ ? / Grüßt den ${ }_{[0]}$ Major $_{[0]} \operatorname{der}_{[\mathrm{S}]}$ Soldat $_{[\mathrm{S}]}$ ?

Greets the $_{[S]}$ soldier $_{[s]}$ the $_{[0]}$ major $_{[0]}$ ? / Greets the ${ }_{[O]}$ major $_{[O]}$ the $_{[S]}$ soldier $_{[S]}$ ?

(4) Examples of questions that require a 'no' response

Grüßt der $_{[\mathrm{S}]}$ Major $_{[\mathrm{S}]}$ den $_{[\mathrm{O}]}$ Soldat $_{[\mathrm{O}]}$ ? / Rügt der ${ }_{[\mathrm{S}]}$ Soldat $_{[\mathrm{S}]}$ den $_{[\mathrm{O}]}$ Major $_{[0]}$ ?

Greets the $_{[S]}$ major $_{[S]}$ the $_{[0]}$ soldier $_{[0]}$ ? / Blames the $e_{[S]}$ soldier $_{[S]}$ the $_{[0]}$ major $_{[O]}$ ?

S: subject, O: object.

\subsubsection{Neuropsychological assessment - General cognitive functioning}

Furthermore, to ensure that the results in the prosody task were not due to general cognitive deficits, we applied a brief battery of standard neuropsychological tests. This battery assessed the ability to store and rehearse verbal and spatial contents using the digit-span and block-span test of the Wechsler Memory Scale (WMS-R; Wechsler, 1987), the ability to focus attention using the $\mathrm{d} 2$ test that requires speeded detection of target symbols amongst distractors (Brickenkamp, 1994), and visuo-spatial reasoning using the third subtest of the Leistungsprüfsystem (LPS-3) that requires identification of rule violations in series of symbols (Horn, 1983). At the outset as well as post-surgically, the patient's performance was within normal average-low to above-average range relative to the age matched normative samples provided by the standard neuropsychological tests (pre-/post-surgical percentile rank of forward digit-span: 35/35; backward digit-span: not assessed; forward block-span: 93/32; backward block-span: 23/23; LPS-3: 69/93; d2: 34/54; standard diagnostic cutoffs of normal average performance: 16-84). 


\subsection{Data analysis}

\subsubsection{Diffusion MRI data}

Diffusion MRI data were analyzed using LIPSIA (Max Planck Institute for Human Cognitive and Brain Sciences, Leipzig, Germany), and FSL (FMRIB, University of Oxford, UK, www.fmrib.ox.ac.uk/fsI). First, $\mathrm{T}_{1^{-}}$ weighted structural scans were reoriented to the sagittal intercommissural plane and the brain was segmented in LIPSIA. A trimmed brain mask was created by shrinking the inner skull surface by $7 \mathrm{~mm}$ to later reduce noisy endpoints of streamlines. Then, motion correction parameters for the 60 diffusionweighted images were computed based on the 7 (b0) reference images distributed over the entire sequence using rigid-body transformations implemented in FSL. Interpolated motion correction parameters were combined with a global registration to the $T_{1}$-anatomy and applied to all 60 volumes that were resampled to an isotropic resolution of $1.7 \mathrm{~mm}$. The gradient direction for each volume was corrected using the rotation parameters. Finally, the diffusion tensor, the three eigenvectors, and the fractional anisotropy (FA) value were computed for each voxel.

The diffusion tensor image was used for full-brain deterministic fiber tracking using an in-house implementation of the tensor deflection algorithm (Lazar et al., 2003) in all voxels of the trimmed brain mask. Together with a lowered FA threshold of 0.075 , this algorithm allowed for robust tracking in areas of low anisotropy (i.e., the edema) while excluding the tumor area and the ventricles. Lowering the FA threshold without any additional processing might, however, introduce false positive connections. To eliminate spurious streamlines while retaining the bundles of interest, tracking was performed in an adapted two-step approach. First, right AF/SLF and posterior CC were selected from the full-brain tracking using inclusion and exclusion masks. The volume of the extracted bundle was computed by selecting all voxels that were crossed by at least two streamlines. In this way, spurious single streamlines were excluded. Inclusion masks for right AF/SLF were placed in the temporal lobe near the temporoparietal junction and in the posterior frontal lobe (Catani, Jones, \& Ffytche, 2005; Mori, 2007). Streamlines crossing the extreme capsule and the thalamus were excluded. Inclusion masks for posterior CC were placed in isthmus and splenium of the CC and right posterior temporal lobe. Streamlines reaching parietal or occipital lobe were excluded. Masks were individually adapted for every participant. In the second step, the extracted bundle volume was dilated by $1 \mathrm{~mm}$ and streamline tracking was performed again, restricted to this volume. Then, the same inclusion and exclusion masks as described above were applied again and the final bundle was extracted. 
The same procedure was applied to define left AF/SLF and right IFOF, as contralateral dorsal and ipsilateral ventral control tracts, respectively. Masks for left AF/SLF were analogous to those used in the right hemisphere. Inclusion masks for the right IFOF were placed in right IFG and right angular gyrus (Makris \& Pandya, 2009). Streamlines targeting areas dorsal of angular gyrus or crossing right AF/SLF or the corpus callosum were excluded.

To assess recovery of FA, mean FA values of right AF/SLF and posterior CC were extracted from pre- and post-surgical scans, limited to the central horizontal parts of the extracted bundle volumes, i.e., deep white matter (see borders in Figure 1A and B) where tractography is most robust and reproducible and FA values are less affected by partial volume effects than in the fanning ends of the bundle. To demonstrate general test/re-test stability of FA (compare green squares and diamonds in Figure 1C), mean FA values of left AF/SLF and right IFOF were extracted in the same way, also excluding the fanning ends of the tract. The IFOF runs ventral of the edema but it was made sure that the chosen segment fully covered the extent of the edema along its y-dimension. The patient's FA values were statistically compared to those of the $\mathrm{HC}$ by means of bootstrapped confidence intervals (see section 2.4.2).

\subsubsection{Behavioral data}

Prosody perception and syntax processing abilities were quantified as \%correct responses. Cognitive abilities were compared based on raw test scores (Table 2; raw scores rather than percentile ranks were used for statistical comparison to preserve original distribution and differences between scale units; Thorndike \& Thorndike-Christ, 2013). Change scores were calculated for each test by subtracting presurgical ( $1^{\text {st }}$ session) from post-surgical $\left(2^{\text {nd }}\right.$ session) performance measures. To specifically compare performance changes in the two matched language tasks-prosody vs. syntax-the difference between the two change scores ([Prosody $y_{\text {post }}-$ Prosody $\left._{\text {pre }}\right]-\left[\right.$ Syntax $_{\text {post }}-$ Syntax $\left.\left._{\text {pre }}\right]\right)$ was calculated. For statistical comparison of patient's and HC's data, we chose a non-parametric bootstrapping approach as in Meyer et al. (2014) to account for the limited number of HC and the resulting violations of the sphericity assumption (Mauchly, 1940). We generated two-tailed 95\% confidence intervals (Cls) based on 10000 random draws from the values of HC (Efron, 1979) as implemented in MATLAB (The MathWorks, Inc., Natick, MA, USA). Patient's scores were considered significantly different from those of HC when they were outside these bootstrapped Cls. HC's performance in $1^{\text {st }}$ and $2^{\text {nd }}$ session was compared by means of two-tailed $t$-tests for paired samples. 


\section{Results}

\subsection{Anatomy and diffusion MRI data}

Post-surgically, the tumor was fully resected with no remaining cavity, and the edema was entirely reabsorbed (Figure 1B; see also Supplementary Figure 2). As shown in Figure 1C and Table 2, the patient's right AF/SLF showed significantly reduced mean FA in the pre-surgical session (green square; $M$ $=0.238$ ) compared to $H C$ (green circles; $M \pm S D=0.481 \pm 0.010$ ) that recovered to near-normal values after surgery (green diamond; $M=0.421$ ), although it remained below HC's mean FA values. Similarly, the patient's posterior CC connecting the temporal lobes showed significantly reduced pre-surgical FA $(M=0.470)$ compared to $H C(M \pm S D=0.619 \pm 0.178)$ that fully recovered after surgery $(M=0.643)$. In turn, the patient's left AF/SLF showed nearly identical FA values in the pre- $(M=0.457)$ and post-surgical session $(M=0.459)$, both not significantly different from mean FA in $H C(M \pm S D=0.454 \pm 0.013)$. Likewise, the patient's right IFOF showed stable FA values in the pre- $(M=0.478)$ and post-surgical scan $(M=0.454)$ that were both within the bounds of $\mathrm{HC}^{\prime}$ s bootstrapped $\mathrm{Cl}(M \pm S D=0.472 \pm 0.029$; for statistical values, see Table 2).

Table 2. Statistical comparison of fractional anisotropy in patient and healthy controls.

\begin{tabular}{|c|c|c|c|c|c|c|c|}
\hline \multirow{2}{*}{ Fiber tract } & \multicolumn{2}{|c|}{ Healthy Controls } & \multicolumn{2}{|c|}{$95 \% \mathrm{Cl}$} & \multicolumn{3}{|c|}{ Patient } \\
\hline & $M$ & SEM & Lower & Upper & Pre & Post & Change \\
\hline Right AF/SLF & 0.481 & 0.010 & 0.476 & 0.491 & 0.238 & 0.421 & +0.183 \\
\hline Posterior CC & 0.619 & 0.178 & 0.596 & 0.648 & 0.470 & 0.643 & +0.173 \\
\hline Left AF/SLF & 0.454 & 0.013 & 0.446 & 0.464 & 0.457 & 0.459 & +0.002 \\
\hline Right IFOF & 0.472 & 0.029 & 0.453 & 0.494 & 0.478 & 0.454 & -0.024 \\
\hline
\end{tabular}

AF/SLF: arcuate/superior longitudinal fascicle, CC: corpus callosum, IFOF: inferior fronto-occipital fascicle. Bold values represent significant lower fractional anisotropy in patient than healthy controls.

\subsection{Behavioral data}

In the prosody task, our patient showed a performance increase from pre- to post-surgical session $(+12.50 \%)$ that was significantly stronger than the nominal performance change in $\mathrm{HC}(M \pm S D=2.08 \% \pm$ 2.33\%; see Figure 1D and Table 3 for statistical details). Pre-surgically, the patient showed significantly lower performance $(65.63 \%)$ than $\mathrm{HC}(M \pm S D=75.11 \% \pm 3.91 \%)$, whereas his performance reached normal levels in the post-surgical session (78.13\%; $\mathrm{HC}: M \pm S D=77.19 \% \pm 5.41 \%)$. $\mathrm{HC}$ performed equally well across the two sessions (paired samples $t$-test: $t(9)=-0.89, p>.395$ ). 
In the non-prosodic syntax task, our patient showed stable performance across sessions (pre: 76.67\%; post: $73.3 \%)$, similar to $H C\left(1^{\text {st }}: M \pm S D=79.22 \% \pm 3.36 \% ; 2^{\text {nd }}: 75.33 \% \pm 4.99 \%\right)$. Performance did not differ between patient and $\mathrm{HC}$, neither pre- nor post-surgically nor in terms of performance change over time (see Table 3 for $\mathrm{Cl}$ 's). HC showed no performance differences between the two sessions $(t(9)=1.29, p>.229$; see Figure $1 D)$.

The comparison of the change scores between the two tasks revealed that the patient's pre-postsurgical performance gain was stronger (by $15.84 \%$ ) in the prosody than in the syntax task, which was significantly more than in $\mathrm{HC}(\mathrm{Cl}: 0.15 \%-12.99 \%)$. Note that this result cannot be due to general differences in task difficulty or ceiling and floor effects. $\mathrm{HC}^{\prime}$ s results confirmed that the two tasks were perfectly matched in difficulty (no significant main effect of TASK in a repeated-measures ANOVA with factors TASK and SESSION: $F(1,9)=0.07, p>.791$ ) and were well above chance level (one-sample $t$-tests against $50 \%$ per session and task: $t^{\prime} s>5.02, p^{\prime} s<.002$ ) and well below ceiling (one-sample $t$-tests against $100 \%$ per session and task: $\left.t^{\prime} s<-4.21, p^{\prime} s<.003\right)$.

Likewise, the patient's performance gain in the prosody task cannot be explained by a putative recovery of general cognitive functions. In none of the cognitive tasks, our patient showed a pattern of presurgical deficit and post-surgical recovery (as observed in the prosody task). In the span tests, the patient showed perfectly stable (forward digit-span, backward block-span) or post-surgically decreased (instead of increased) performance (forward block-span). In the attention task (d2), patient's performance increased over time, however, to a similar extent as in $\mathrm{HC}(t(9)=-7.64, p<.001)$, i.e., denoting a general learning effect rather than recovery. Overall, in both the attention task and the span tests, our patient scored mostly slightly below HC, constantly across both sessions, making it unlikely that these abilities contributed to performance recovery in the prosody test. Only exception was the spatial-reasoning task (LPS-3). Here, the patient showed a pre-post-surgical performance increase that was significantly stronger than in $\mathrm{HC}$, although his pre-surgical performance did not significantly differ from HC's scores. This finding is compatible with right dorsal pathway involvement in spatial relational reasoning (Krawczyk, 2012; Shokri-Kojori, Motes, Rypma, \& Krawczyk, 2012; Watson \& Chatterjee, 2012). 
Table 3. Statistical comparison of behavioral results of patient and healthy controls in the two sessions.

\begin{tabular}{|c|c|c|c|c|c|c|}
\hline \multirow{2}{*}{ Test } & \multicolumn{2}{|c|}{ Healthy Controls } & \multicolumn{2}{|c|}{$95 \% \mathrm{Cl}$} & \multicolumn{2}{|c|}{ Patient } \\
\hline & $M$ & SEM & Lower & Upper & Score & Direction \\
\hline
\end{tabular}

Critical tests (\% correct)

Prosody perception

$\begin{array}{lrrrrrr}1^{\text {st }} \text { session } & 75.11 & 3.91 & 68.23 & 82.82 & 65.63 & - \\ 2^{\text {nd }} \text { session } & 77.19 & 5.41 & 67.25 & 87.12 & 78.13 & = \\ \text { difference }\left(2^{\text {nd }}-1^{\text {st }} \text { session }\right) & 2.08 & 2.33 & -2.61 & 6.04 & \mathbf{1 2 . 5 0} & +\end{array}$

Syntax processing

$\begin{array}{lrrrrrl}1^{\text {st }} \text { session } & 79.22 & 3.36 & 73.33 & 85.67 & 76.67 & = \\ 2^{\text {nd }} \text { session } & 75.33 & 4.99 & 66.17 & 84.45 & 73.33 & = \\ \text { difference }\left(2^{\text {nd }}-1^{\text {st }} \text { session }\right) & -3.89 & 3.01 & -9.17 & 2.22 & -3.34 & =\end{array}$

General cognitive functions (raw scores)

d2 concentration performance

$\begin{array}{lrrrrrr}1^{\text {st }} \text { session } & 175.20 & 10.30 & 158.00 & 196.20 & 141 & - \\ 2^{\text {nd }} \text { session } & 197.50 & 10.40 & 178.50 & 217.00 & 163 & - \\ \text { difference }\left(2^{\text {nd }}-1^{\text {st }} \text { session }\right) & 22.30 & 2.92 & 17.60 & 28.60 & 22 & =\end{array}$

Digit span forward

$\begin{array}{lllllll}1^{\text {st }} \text { session } & 8.10 & 0.48 & 7.10 & 8.90 & 7 & - \\ 2^{\text {nd }} \text { session } & 8.10 & 0.60 & 7.00 & 9.30 & 7 & = \\ \text { difference }\left(2^{\text {nd }}-1^{\text {st }} \text { session }\right) & 0.00 & 0.49 & -1.00 & 0.80 & 0 & =\end{array}$

\section{Block span backward}

$\begin{array}{lrrrrrrr}1^{\text {st }} \text { session } & 8.40 & 0.58 & 7.20 & 9.40 & \mathbf{7} & - \\ 2^{\text {nd }} \text { session } & 9.60 & 0.67 & 8.10 & 10.60 & \mathbf{7} & - \\ \text { difference }\left(2^{\text {nd }}-1^{\text {st }} \text { session }\right) & 1.20 & 0.53 & 0.40 & 2.40 & \mathbf{0} & -\end{array}$

Block span forward

$\begin{array}{lrlllrl}1^{\text {st }} \text { session } & 8.60 & 0.37 & 8.00 & 9.50 & \mathbf{1 2} & + \\ 2^{\text {nd }} \text { session } & 9.30 & 0.37 & 8.60 & 9.90 & \mathbf{8} & - \\ \text { difference }\left(2^{\text {nd }}-1^{\text {st }} \text { session }\right) & 0.70 & 0.37 & 0.10 & 1.50 & \mathbf{- 4} & -\end{array}$

LPS-3

$\begin{array}{lrrrrrr}1^{\text {st }} \text { session } & 27.50 & 1.92 & 21.80 & 30.00 & 23 & = \\ 2^{\text {nd }} \text { session } & 29.90 & 1.22 & 27.40 & 32.00 & 30 & = \\ \text { difference }\left(2^{\text {nd }}-1^{\text {st }} \text { session }\right) & 2.40 & 1.38 & 0.10 & 5.50 & \mathbf{7} & +\end{array}$

Bold values represent significant differences between patient and healthy controls. Symbols indicate whether patient's performance (change) was higher (+), similar to (=) or lower than (-) in controls.

\section{Discussion}

Following the hypotheses that right dorsal (Sammler et al., 2015) and posterior transcallosal fiber tracts (Friederici et al., 2007; Sammler et al., 2010) support prosodic structure building, and that vasogenic 
edemas can induce reversible deficits when infiltrating white matter tracts (Bizzi et al., 2012; Gierhan et al., 2012), we tested an edema patient on his abilities to process linguistic prosody, both before and after edema treatment and neurosurgery. Before treatment, i.e., when the edema was infiltrating and compressing right $\mathrm{AF} / \mathrm{SLF}$ and posterior $\mathrm{CC}$, the patient presented deficits in recognizing irregular prosody that mismatched syntactic structure. Reabsorption of the edema and resection of the benign meningioma were associated with a reversal of these deficits. This behavioral recovery was accompanied by increased average FA values in right AF/SLF and posterior CC, while FA was constant in left AF/SLF and right IFOF. Neither short term and working memory nor syntactic comprehension exhibited a similar pre-to-post-surgical performance gain. Consequently, the reversal of the prosodic deficit cannot be due to a nonspecific recovery of cognitive or verbal abilities. Rather, the findings invite the discussion of causal involvement of right dorsal and posterior transcallosal fiber tracts in the processing of prosodic structure.

What remains to be resolved is exactly how these pathways may contribute to the processing of prosodic information. In cognitive terms, it seems plausible to think of prosodic structure building as a hierarchical multi-step process with information passing through consecutive stages of basic acoustic analyses, higher-level auditory grouping and integration with syntactic and semantic information (for similar multi-step models of emotional prosody, see Brück, Kreifelts, \& Wildgruber, 2011; Kotz \& Paulmann, 2011; Schirmer \& Kotz, 2006; Wildgruber, Ackermann, Kreifelts, \& Ethofer, 2006). Intra- and inter-hemispheric pathways secure rapid and bidirectional information exchange within and between these stages represented in distributed neural networks. Although the present study cannot dissociate the functions supported by right dorsal and transcallosal pathways (because both of them were infiltrated by the edema), models of auditory, speech and language processing (Bornkessel-Schlesewsky, Schlesewsky, Small, \& Rauschecker, 2015; Friederici, 2011; Friederici \& Alter, 2004; Hickok \& Poeppel, 2007; Rauschecker \& Scott, 2009) may lend a basis to start reflecting upon possible mechanisms, as will be done in the remainder of the text. Three capacities should be considered: (i) the time-sensitive tracking of prosodic features and (ii) their grouping into higher order structures, and (iii) the integration of syntactic and prosodic information.

\subsection{Right dorsal pathways - time-sensitive tracking and structuring of prosodic features}

The decision upon a sentence's prosodic form and regularity, as in the present study, requires processing of auditory-prosodic cues, e.g., the recognition of pitch contours as either rising (here: 
regular) or falling (irregular). This requirement may tap into the capacity of dorsal pathways to track auditory information-including pitch-over time. In its accepted role to map sound to articulation (Hickok \& Poeppel, 2007; Saur et al., 2008), dorsal pathways are typically assumed to host auditorymotor loops that continuously monitor the sound and pitch of one's own vocalizations during speech production (Guenther \& Vladusich, 2012; Hickok, 2012; Houde \& Chang, 2015; Zarate, 2013). The same auditory-motor system in reversed processing mode has been proposed to serve speech perception (Hickok, Houde, \& Rong, 2011; Rauschecker, 2011), including perception of prosodic contour (Sammler et al., 2015) and discrimination of vocal pitch (D'Ausilio, Bufalari, Salmas, Busan, \& Fadiga, 2011). Altogether, it seems that dorsal connections between auditory temporal and (pre)motor areas could provide a basic computational building block necessary to track prosodic pitch contours over time, that may have been temporarily disrupted in our patient.

From a higher-order linguistic point of view, successful parsing of prosodic structure involves the segmentation of sentence-level prosodic information into constituent elements, e.g., intonational phrases (Nespor \& Vogel, 1986; Selkirk, 1984). This requirement resonates with recent proposals that the dorsal stream may identify basic prosodic units (e.g., prosodic words) and combine them into successively larger linguistic chunks over time (e.g., intonational phrases; Bornkessel-Schlesewsky \& Schlesewsky, 2013). Right AF/SLF involvement in the structuring of auditory pitch information in music (Loui, Alsop, \& Schlaug, 2009; Peretz, 2016)(but see Chen et al., 2015) may provide cross-domain support for this idea. To date, only little is known about the neuroanatomical bases of sentence-level prosodic structure building. However, several findings are compatible with fronto-temporal information exchange during prosodic phrasing (Geiser, Zaehle, Jancke, \& Meyer, 2008; Ischebeck, Friederici, \& Alter, 2008; Strelnikov, Vorobyev, Chernigovskaya, \& Medvedev, 2006)(but see den Ouden et al., 2016). Whether or not the fronto-temporal information exchange happens via a dorsal posterior temporal to inferior frontal pathway, as possibly suggested by our patient's performance, is an interesting topic for future research.

Irrespective of whether dorsal white matter tracts constitute building blocks for basic pitch monitoring or advanced prosodic structuring (or both in interaction), their computational characteristics may be described in terms of internal (forward) models that serve to predict forthcoming sensory events (here: the to-be-perceived pitch contour) on the basis of previous input (Bornkessel-Schlesewsky \& Schlesewsky, 2013; Rauschecker, 2011). Notably, these predictions are likely to arise from both prosodic 
and non-prosodic priors-including continuity of pitch contour and prosodic phrase but also syntactic structure, as will be discussed in the following.

\subsection{Posterior transcallosal pathways - syntax-prosody interface}

Syntactic structure guides prosodic parsing (Buxó-Lugo \& Watson, 2016; Cole et al., 2010; Cutler et al., 1997) and vice versa (Lehiste, 1973; Snedeker \& Trueswell, 2003) and the posterior corpus callosum has been proposed as the relevant interface (Friederici et al., 2007; Sammler et al., 2010). The present task drew on this interface by violating prosodic expectancies that were established through syntactic structure (see Methods). The patient's sentence-level syntactic processing was arguably intact in both sessions as indicated by his unimpaired performance in the non-prosodic syntax task. Yet, online syntactic processes may have no longer triggered the build-up of prosodic expectancies because of the temporary disruption of the necessary crosstalk between syntax and prosody processing streams. This transient deficit would mark a further case for the relevance of the posterior CC for information exchange between the temporal lobes in syntax-prosody alignment.

Taken together, our patient's performance pattern may be interpreted as temporary deficit in tracking and/or predicting prosodic contour at lower and/or higher linguistic levels. Although we cannot isolate the relative contribution of intra- and inter-hemispheric fiber tracts in the present case, both the capacity of dorsal pathways to process auditory-prosodic information in a time-sensitive manner (Bornkessel-Schlesewsky \& Schlesewsky, 2013; Frühholz \& Grandjean, 2013; Kreiner \& Eviatar, 2014; Rauschecker, 2011) and the role of transcallosal pathways in interfacing prosody and syntax may satisfy crucial requirements for prosodic parsing: the analysis how prosodic information evolves over time and relative to concurrent syntactic information.

\subsection{Focality of edema-induced disruptions}

Vasogenic edemas are extracellular edemas; they spread along white matter tracts and are assumed to temporarily disturb information flow along these tracts (Bizzi et al., 2012; Gierhan et al., 2012). It is, however, difficult to reliably estimate the extent of the affected region. First, the edema invaded the posterior CC as well as several dorsal sub-pathways, including those supporting visuo-spatial attention and relational integration (Krawczyk, 2012; Shokri-Kojori et al., 2012; C. E. Watson \& Chatterjee, 2012) such as right SLF III (Thiebaut de Schotten, Dell'Acqua, et al., 2011). The latter may account for the patient's post-surgical performance increase in visuo-spatial reasoning. A second obvious concern is that 
space-occupying edemas may compromise not only fiber tracts but may also reduce functionality of surrounding grey matter through compression, i.e., of right parietal areas in the present study. Prevailing models of prosody perception do not typically include the parietal lobe (Brück et al., 2011; Friederici \& Alter, 2004; Schirmer \& Kotz, 2006; Wildgruber et al., 2006; Wildgruber, Ethofer, Grandjean, \& Kreifelts, 2009; Witteman, Van Heuven, \& Schiller, 2012); nevertheless, right inferior parietal activations (BA 40/7) have been occasionally reported in prosody studies (Belyk \& Brown, 2014; Merrill et al., 2012) and were proposed to reflect rehearsal-based working memory processes for prosodic pitch contours (Kreitewolf et al., 2014; Perrone-Bertolotti et al., 2013). The present study cannot discriminate between neural mass effects and changes in white matter conductivity. Yet, it is notable that the recovery of prosodic deficits was accompanied by increased average FA values in those tracts that had passed through the edema before surgery. This relationship between recovered diffusivity values and recovered cognitive functions makes it likely that white matter connectivity contributed to the behavioral effects. The potential contribution of IPL to the observed effects can be probed in future studies with healthy participants by means of transcranial magnetic stimulation.

\section{Conclusion}

In summary, the present case study provides new insights into right dorsal and posterior transcallosal pathway functions in auditory language comprehension by demonstrating that temporary edemainduced dysfunction of right AF/SLF and posterior CC perturbs sentence-level prosody perception. The sensitivity of dorsal pathways to temporal dynamics of auditory information may constitute the decisive computational feature that provides the dorsal pathways with the capacity to track, predict and/or evaluate prosodic contour over time. Future research should look into potential divisions of labor between different dorsal sub-pathways and their interaction. Assuming functional parallels to left dorsal pathways, posterior temporal to premotor connections might be particularly suited to track pitch over time by virtue of the time-sensitivity of the motor system (Houde \& Chang, 2015; Rauschecker, 2011). Posterior temporal to inferior frontal connections, in turn, might be involved in prosodic structure building by virtue of the combinatorial capacities of IFG (Friederici, 2011; Koelsch, 2005) and a linkage with non-prosodic syntactic information provided by the left hemisphere via the posterior corpus callosum (Friederici et al., 2007; Sammler et al., 2010). 


\section{Statement of Significance}

Dorsal and ventral pathways in the left hemisphere play established roles in syntactic parsing and semantic comprehension, while pathways for linguistic prosody perception remain little explored. The present single case lesion study suggests a causal role of right dorsal pathways in prosodic structure building that further depends on the integrity of the posterior corpus callosum to interact with concurrent syntactic information.

\section{Acknowledgements}

We thank Johanna Reichert and Katharina Fath for help with testing healthy controls. This research was funded by the Max Planck Society.

\section{References}

Baum, S. R., \& Pell, M. D. (1999). The neural bases of prosody: Insights from lesion studies and neuroimaging. Aphasiology, 13(8), 581-608.

Belyk, M., \& Brown, S. (2014). Perception of affective and linguistic prosody: An ALE meta-analysis of neuroimaging studies. Social Cognitive and Affective Neuroscience, 9(9), 1395-403.

Bizzi, A., Nava, S., Ferre, F., Castelli, G., Aquino, D., Ciaraffa, F., ... Piacentini, S. (2012). Aphasia induced by gliomas growing in the ventrolateral frontal region: Assessment with diffusion MR tractography, functional MR imaging and neuropsychology. Cortex, 48(2), 255-272.

Blumstein, S., \& Cooper, W. E. (1974). Hemispheric processing of intonation contours. Cortex, 10(2), 146-158.

Bornkessel-Schlesewsky, I., \& Schlesewsky, M. (2013). Reconciling time, space and function: A new dorsal-ventral stream model of sentence comprehension. Brain and Language, 125(1), 60-76.

Bornkessel-Schlesewsky, I., Schlesewsky, M., Small, S. L., \& Rauschecker, J. P. (2015). Neurobiological roots of language in primate audition: Common computational properties. Trends in Cognitive Sciences, 19(3), 142-150.

Brickenkamp, R. (1994). Test d2. Göttingen: Hogrefe.

Brück, C., Kreifelts, B., \& Wildgruber, D. (2011). Emotional voices in context: A neurobiological model of multimodal affective information processing. Physics of Life Reviews, 8(4), 383-403.

Buxó-Lugo, A., \& Watson, D. G. (2016). Evidence for the influence of syntax on prosodic parsing. Journal of Memory and Language, 90, 1-13.

Catani, M., Jones, D. K., \& Ffytche, D. H. (2005). Perisylvian language networks of the human brain. Annals of Neurology, 57(1), 8-16.

Chen, J. L., Kumar, S., Williamson, V. J., Scholz, J., Griffiths, T. D., \& Stewart, L. (2015). Detection of the arcuate fasciculus in congenital amusia depends on the tractography algorithm. Frontiers in Psychology, 6, 1-11.

Cole, J. (2015). Prosody in context: A review. Language, Cognition and Neuroscience, 30(1-2), 1-31. 
Cole, J., Mo, Y., \& Baek, S. (2010). The role of syntactic structure in guiding prosody perception with ordinary listeners and everyday speech. Language and Cognitive Processes, 25(7-9), 1141-1177.

Cooper, W. E., \& Paccia-Cooper, J. (1980). Syntax and speech. Cambridge, MA: Harvard University Press.

Cutler, A., Dahan, D., \& Van Donselaar, W. (1997). Prosody in the comprehension of spoken language: A literature review. Language and Speech, 40, 141-201.

D’Ausilio, A., Bufalari, I., Salmas, P., Busan, P., \& Fadiga, L. (2011). Vocal pitch discrimination in the motor system. Brain and Language, 118(1-2), 9-14.

den Ouden, D.-B., Dickey, M. W., Anderson, C., \& Christianson, K. (2016). Neural correlates of earlyclosure garden-path processing: Effects of prosody and plausibility. Quarterly Journal of Experimental Psychology, 69(5), 926-949.

Domahs, U., Klein, E., Huber, W., \& Domahs, F. (2013). Good, bad and ugly word stress - fMRI evidence for foot structure driven processing of prosodic violations. Brain and Language, 125(3), 272-282.

Duffau, H., Leroy, M., \& Gatignol, P. (2008). Cortico-subcortical organization of language networks in the right hemisphere: An electrostimulation study in left-handers. Neuropsychologia, 46(14), 31973209.

Eckstein, K., \& Friederici, A. D. (2006). It's early: Event-related potential evidence for initial interaction of syntax and prosody in speech comprehension. Journal of Cognitive Neuroscience, 18(10), 16961711.

Efron, B. (1979). Bootstrap methods: Another look at the jackknife. The Annals of Statistics, 7, 1-26.

Fernández-Miranda, J. C., Wang, Y., Pathak, S., Stefaneau, L., Verstynen, T., \& Yeh, F.-C. (2015). Asymmetry, connectivity, and segmentation of the arcuate fascicle in the human brain. Brain Structure \& Function, 220(3), 1665-1680.

Forkel, S. J., Thiebaut de Schotten, M., Dell'Acqua, F., Kalra, L., Murphy, D. G., Williams, S. C., \& Catani, M. (2014). Anatomical predictors of aphasia recovery: A tractography study of bilateral perisylvian language networks. Brain, 137, 2027-2039.

Fridriksson, J., den Ouden, D.-B., Hillis, A. E., Hickok, G., Rorden, C., Basilakos, A., ... Bonilha, L. (2018). Anatomy of aphasia revisited. Brain.

Friederici, A. D. (2011). The brain basis of language processing: From structure to function. Physiological Reviews, 91(4), 1357-1392.

Friederici, A. D. (2012). The cortical language circuit: From auditory perception to sentence comprehension. Trends in Cognitive Sciences, 16(5), 262-268.

Friederici, A. D., \& Alter, K. (2004). Lateralization of auditory language functions: A dynamic dual pathway model. Brain and Language, 89(2), 267-276.

Friederici, A. D., \& Gierhan, S. M. E. (2013). The language network. Current Opinion in Neurobiology, 23(2), 250-254.

Friederici, A. D., von Cramon, D. Y., \& Kotz, S. A. (2007). Role of the corpus callosum in speech comprehension: Interfacing syntax and prosody. Neuron, 53(1), 135-145.

Frühholz, S., \& Grandjean, D. (2013). Processing of emotional vocalizations in bilateral inferior frontal cortex. Neuroscience and Biobehavioral Reviews, 37, 2847-2855.

Frühholz, S., Gschwind, M., \& Grandjean, D. (2015). Bilateral dorsal and ventral fiber pathways for the processing of affective prosody identified by probabilistic fiber tracking. Neurolmage, 109, 27-34.

Gandour, J. T., Tong, Y. X., Wong, D., Talavage, T., Dzemidzic, M., Xu, Y. S., ... Lowe, M. (2004). Hemispheric roles in the perception of speech prosody. Neuroimage, 23(1), 344-357. 
Geiser, E., Zaehle, T., Jancke, L., \& Meyer, M. (2008). The neural correlate of speech rhythm as evidenced by metrical speech processing. Journal of Cognitive Neuroscience, 20(3), 541-552.

Gierhan, S. M. E. (2013a). Brain networks for language: Anatomy and functional roles of neural pathways supporting language comprehension and repetition. MPI Series (Vol. 144). Leipzig: Max Planck Institute for Human Cognitive and Brain Sciences.

Gierhan, S. M. E. (2013b). Connections for auditory language in the human brain. Brain and Language, $127,205-221$.

Gierhan, S. M. E., Rhone, P., Anwander, A., Jost, I., Frydrychowicz, C., Hoffmann, K.-T., ... Friederici, A. D. (2012). Tracking the language pathways in edema patients: Preliminary results. In U. Goltz, M. Magnor, H.-J. Appelrath, H. Mathies, W.-T. Balke, \& L. Wolf (Eds.), Informatik 2012 (pp. 11021111). Bonn: Köllen Verlag.

Giraud, A. L., Kleinschmidt, A., Poeppel, D., Lund, T. E., Frackowiak, R. S., \& Laufs, H. (2007). Endogenous cortical rhythms determine cerebral specialization for speech perception and production. Neuron, 56(6), 1127-1134.

Glasser, M. F., \& Rilling, J. K. (2008). DTI tractography of the human brain's language pathways. Cerebral Cortex, 18(11), 2471-2482.

Griffiths, J. D., Marslen-Wilson, W. D., Stamatakis, E. A., \& Tyler, L. K. (2013). Functional organization of the neural language system: Dorsal and ventral pathways are critical for syntax. Cerebral Cortex, 23(1), 139-147.

Guenther, F. H., \& Vladusich, T. (2012). A neural theory of speech acquisition and production. Journal of Neurolinguistics, 25(5), 408-422.

Hellbernd, N., \& Sammler, D. (2016). Prosody conveys speaker's intentions: Acoustic cues for speech act perception. Journal of Memory and Language, 88, 70-86.

Hickok, G. (2012). Computational neuroanatomy of speech production. Nature Reviews Neuroscience, 13(2), 135-145.

Hickok, G., Houde, J. F., \& Rong, F. (2011). Sensorimotor integration in speech processing: Computational basis and neural organization. Neuron, 69(3), 407-422.

Hickok, G., \& Poeppel, D. (2007). The cortical organization of speech processing. Nature Reviews Neuroscience, $8(5), 393-402$.

Hofer, S., \& Frahm, J. (2006). Topography of the human corpus callosum revisited - Comprehensive fiber tractography using diffusion tensor magnetic resonance imaging. Neurolmage, 32(3), 989-994.

Horn, W. (1983). Leistungsprüfsystem. Göttingen: Hogrefe.

Houde, J. F., \& Chang, E. F. (2015). The cortical computations underlying feedback control in vocal production. Current Opinion in Neurobiology, 33, 174-181.

Huang, H., Zhang, J., Jiang, H., Wakana, S., Poetscher, L., Miller, M. I., ... Mori, S. (2005). DTI tractography based parcellation of white matter: Application to the mid-sagittal morphology of corpus callosum. Neurolmage, 26(1), 195-205.

Ischebeck, A. K., Friederici, A. D., \& Alter, K. (2008). Processing prosodic boundaries in natural and hummed speech: An fMRI study. Cerebral Cortex, 18(3), 541-552.

Jamison, H. L., Watkins, K. E., Bishop, D. V. M., \& Matthews, P. M. (2006). Hemispheric specialization for processing auditory nonspeech stimuli. Cerebral Cortex, 16(9), 1266-1275.

Johnsrude, I. S., Penhune, V. B., \& Zatorre, R. J. (2000). Functional specificity in the right human auditory cortex for perceiving pitch direction. Brain, 123, 155-163.

Kellmeyer, P., Ziegler, W., Peschke, C., Juliane, E., Schnell, S., Baumgaertner, A., ... Saur, D. (2013). 
Fronto-parietal dorsal and ventral pathways in the context of different linguistic manipulations. Brain and Language, 127(2), 241-250.

Koelsch, S. (2005). Neural substrates of processing syntax and semantics in music. Current Opinion in Neurobiology, 15(2), 207-212.

Kotz, S. A., \& Paulmann, S. (2011). Emotion, language, and the brain. Language and Linguistics Compass, 5(3), 108-125.

Krawczyk, D. C. (2012). The cognition and neuroscience of relational reasoning. Brain Research, 1428, 13-23.

Kreiner, H., \& Eviatar, Z. (2014). The missing link in the embodiment of syntax: Prosody. Brain and Language, 137, 91-102.

Kreitewolf, J., Friederici, A. D., \& von Kriegstein, K. (2014). Hemispheric lateralization of linguistic prosody recognition in comparison to speech and speaker recognition. Neurolmage, 102, 332-344.

Kümmerer, D., Hartwigsen, G., Kellmeyer, P., Glauche, V., Mader, I., Klöppel, S., ... Saur, D. (2013). Damage to ventral and dorsal language pathways in acute aphasia. Brain, 136(2), 619-629.

Kyong, J. S., Scott, S. K., Rosen, S., Howe, T. B., Agnew, Z. K., \& McGettigan, C. (2014). Exploring the roles of spectral detail and intonation contour in speech intelligibility: An FMRI study. Journal of Cognitive Neuroscience, 26(8), 1748-1763.

Ladd, D. R. (2008). Intonational phonology. Cambridge: Cambridge University Press.

Lazar, M., Weinstein, D. M., Tsuruda, J. S., Hasan, K. M., Arfanakis, K., Meyerand, M. E., ... Alexander, A. L. (2003). White matter tractography using diffusion tensor deflection. Human Brain Mapping, 18(4), 306-321.

Lehiste, I. (1973). Phonetic disambiguation of syntactic ambiguity. Glossa, 7(2), 107-121.

Loui, P., Alsop, D., \& Schlaug, G. (2009). Tone deafness: A new disconnection syndrome? The Journal of Neuroscience, 29(33), 10215-10220.

Makris, N., \& Pandya, D. N. (2009). The extreme capsule in humans and rethinking of the language circuitry. Brain Structure \& Function, 213(3), 343-358.

Mauchly, J. W. (1940). Significance test for sphericity of a normal n-variate distribution. The Annals of Mathematical Statistics, 11, 204-209.

McGettigan, C., \& Scott, S. K. (2012). Cortical asymmetries in speech perception: what's wrong, what's right and what's left? Trends in Cognitive Sciences, 16(5), 269-276.

Meixensberger, J., \& Jaeger, M. (2005). Relevance of ICP and ptiO(2) for indication and timing of decompressive craniectomy in patients with malignant brain edema. Acta Neurochirurgica, 147(9), 952.

Merrill, J., Sammler, D., Bangert, M., Goldhahn, D., Lohmann, G., Turner, R., \& Friederici, A. D. (2012). Perception of words and pitch patterns in song and speech. Frontiers in Psychology, 3, 76.

Meyer, L., Cunitz, K., Obleser, J., \& Friederici, A. D. (2014). Sentence processing and verbal working memory in a white-matter-disconnection patient. Neuropsychologia, 61, 190-196.

Meyer, M., Alter, K., Friederici, A. D., Lohmann, G., \& von Cramon, D. Y. (2002). FMRI reveals brain regions mediating slow prosodic modulations in spoken sentences. Human Brain Mapping, 17(2), 73-88.

Meyer, M., Steinhauer, K., Alter, K., Friederici, A. D., \& von Cramon, D. Y. (2004). Brain activity varies with modulation of dynamic pitch variance in sentence melody. Brain and Language, 89(2), 277289. 
Mori, S. (2007). Introduction to diffusion tensor imaging. Amsterdam: Elsevier.

Nespor, M., \& Vogel, I. (1986). Prosodic phonology. Dordrecht: Foris.

Nishizaki, T., Ozaki, S., Kwak, T., \& Ito, H. (1999). Clinical features and surgical outcome in patients with asymptomatic meningiomas. British Journal of Neurosurgery, 13(1), 52-55.

Obleser, J., Eisner, F., \& Kotz, S. A. (2008). Bilateral speech comprehension reflects differential sensitivity to spectral and temporal features. The Journal of Neuroscience, 28(32), 8116-8123.

Parker, G. J. M., Luzzi, S., Alexander, D. C., Wheeler-Kingshott, C. A. M., Clecarelli, O., \& Ralph, M. A. L. (2005). Lateralization of ventral and dorsal auditory-language pathways in the human brain. Neurolmage, 24(3), 656-666.

Paulmann, S. (2016). The neurocognition of prosody. In G. Hickok \& S. L. Small (Eds.), Neurobiology of Language (pp. 1109-1120). Amsterdam: Elsevier.

Peretz, I. (2016). Neurobiology of congenital amusia. Trends in Cognitive Sciences, 20(11), 857-867.

Perkins, J. M., Baran, J. A., \& Gandour, J. (1996). Hemispheric specialization in processing intonation contours. Aphasiology, 10(4), 343-362.

Perrone-Bertolotti, M., Dohen, M., Lœvenbruck, H., Sato, M., Pichat, C., \& Baciu, M. (2013). Neural correlates of the perception of contrastive prosodic focus in French: A functional magnetic resonance imaging study. Human Brain Mapping, 34(10), 2574-2591.

Peschke, C., Ziegler, W., Eisenberger, J., \& Baumgaertner, A. (2012). Phonological manipulation between speech perception and production activates a parieto-frontal circuit. Neurolmage, 59(1), 788-799.

Pierrehumbert, J., \& Hirshberg, J. (1990). The Meaning of Intonational Contours in the Interpretation of Discourse. In P. R. Cohen Morgan, J., \& Pollack, M. E. (Ed.), Intentions in Communication. Cambridge: MIT Press.

Plante, E., Creusere, M., \& Sabin, C. (2002). Dissociating sentential prosody from sentence processing: Activation interacts with task demands. Neurolmage, 17(1), 401-410.

Poeppel, D. (2003). The analysis of speech in different temporal integration windows: Cerebral lateralization as "asymmetric sampling in time." Speech Communication, 41(1), 245-255.

Powell, H. W. R., Parker, G. J. M., Alexander, D. C., Symms, M. R., Boulby, P. A., Wheeler-Kingshott, C. A. M., ... Duncan, J. S. (2006). Hemispheric asymmetries in language-related pathways: A combined functional MRI and tractography study. Neurolmage, 32(1), 388-399.

Rauschecker, J. P. (2011). An expanded role for the dorsal auditory pathway in sensorimotor control and integration. Hearing Research, 271(1-2), 16-25.

Rauschecker, J. P., \& Scott, S. K. (2009). Maps and streams in the auditory cortex: Nonhuman primates illuminate human speech processing. Nature Neuroscience, 12(6), 718-724.

Rockhill, J., Mrugala, M., \& Chamberlain, M. C. (2007). Intracranial meningiomas: An overview of diagnosis and treatment. Neurosurgical Focus, 23(4), E1.

Sammler, D., Grosbras, M.-H., Anwander, A., Bestelmeyer, P. E. G., \& Belin, P. (2015). Dorsal and ventral pathways for prosody. Current Biology, 25(23), 3079-3085.

Sammler, D., Kotz, S. A., Eckstein, K., Ott, D. V. M., \& Friederici, A. D. (2010). Prosody meets syntax: The role of the corpus callosum. Brain, 133(9), 2643-2655.

Saur, D., Kreher, B. W., Schnell, S., Kümmerer, D., Kellmeyer, P., Vry, M. S., ... Weiller, C. (2008). Ventral and dorsal pathways for language. Proceedings of the National Academy of Sciences of the United States of America, 105(46), 18035-18040.

Scherer, K. R. (1986). Vocal affect expression - A review and a model for future research. Psychological 
Bulletin, 99(2), 143-165.

Schirmer, A., \& Kotz, S. A. (2006). Beyond the right hemisphere: Brain mechanisms mediating vocal emotional processing. Trends in Cognitive Sciences, 10(1), 24-30.

Schlaug, G., Marchina, S., \& Norton, A. (2009). Evidence for plasticity in white-matter tracts of patients with chronic Broca's aphasia undergoing intense intonation-based speech therapy. Annals of the New York Academy of Sciences, 1169, 385-394.

Schönwiesner, M., Rübsamen, R., \& von Cramon, D. Y. (2005). Hemispheric asymmetry for spectral and temporal processing in the human antero-lateral auditory belt cortex. European Journal of Neuroscience, 22(6), 1521-1528.

Selkirk, E. (1984). Phonology and syntax: The relation between sound and structure. Cambridge, MA: MIT Press.

Selkirk, E. (1996). Sentence prosody: intonation, stress, and phrasing. In J. Goldsmith (Ed.), Handbook of phonological theory (pp. 550-569). New York: Blackwell Publishers.

Shipley-Brown, F., Dingwall, W. O., Berlin, C. I., Yeni-Komshian, G., \& Gordon-Salant, S. (1988). Hemispheric processing of affective and linguistic intonation contours in normal subjects. Brain and Language, 33(1), 16-26.

Shokri-Kojori, E., Motes, M. A., Rypma, B., \& Krawczyk, D. C. (2012). The network architecture of cortical processing in visuo-spatial reasoning. Scientific Reports, 2, 411.

Skeide, M. A., Brauer, J., \& Friederici, A. D. (2016). Brain functional and structural predictors of language performance. Cerebral Cortex, 26(5), 2127-2139.

Snedeker, J., \& Trueswell, J. (2003). Using prosody to avoid ambiguity: Effects of speaker awareness and referential context. Journal of Memory and Language, 48(1), 103-130.

Steinmann, S., \& Mulert, C. (2012). Functional relevance of interhemispheric fiber tracts in speech processing. Journal of Neurolinguistics, 25(1), 1-12.

Stokum, J. A., Gerzanich, V., \& Simard, J. M. (2016). Molecular pathophysiology of cerebral edema. Journal of Cerebral Blood Flow and Metabolism, 36(3), 513-538.

Strelnikov, K. N., Vorobyev, V. A., Chernigovskaya, T. V, \& Medvedev, S. V. (2006). Prosodic clues to syntactic processing - A PET and ERP study. Neurolmage, 29(4), 1127-1134.

Tang, C., Hamilton, L. S., \& Chang, E. F. (2017). Intonational speech prosody encoding in the human auditory cortex. Science, 357(6353), 797-801.

Thiebaut de Schotten, M., Dell'Acqua, F., Forkel, S. J., Simmons, A., Vergani, F., Murphy, D. G. M., \& Catani, M. (2011). A lateralized brain network for visuospatial attention. Nature Neuroscience, 14(10), 1245-1246.

Thiebaut de Schotten, M., Ffytche, D. H., Bizzi, A., Dell'Acqua, F., Allin, M., Walshe, M., ... Catani, M. (2011). Atlasing location, asymmetry and inter-subject variability of white matter tracts in the human brain with MR diffusion tractography. Neurolmage, 54(1), 49-59.

Thorndike, R. M., \& Thorndike-Christ, T. M. (2013). Measurement and evaluation in psychology and education (8th ed.). Harlow: Pearson Education Limited.

van Lancker, D. (1980). Cerebral lateralization of pitch cues in the linguistic signal. Papers in Linguistics, 13(2), 201-277.

Vassal, M., Le Bars, E., Moritz-Gasser, S., Menjot, N., \& Duffau, H. (2010). Crossed aphasia elicited by intraoperative cortical and subcortical stimulation in awake patients. Journal of Neurosurgery, 113(6), 1251-1258.

Wagner, M., \& Watson, D. G. (2010). Experimental and theoretical advances in prosody: A review. 
Language and Cognitive Processes, 25(7-9), 905-945.

Watson, C. E., \& Chatterjee, A. (2012). A bilateral frontoparietal network underlies visuospatial analogical reasoning. Neurolmage, 59(3), 2831-2838.

Watson, D. G., \& Gibson, E. (2004). The relationship between intonational phrasing and syntactic structure in language production. Language and Cognitive Processes, 19(6), 713-755.

Wechsler, D. (1987). Wechsler Memory Scale - Revised (WMS-R). San Antonio, CA: Psychological Corporation/Harcourt Brace Jovanovich.

Wildgruber, D., Ackermann, H., Kreifelts, B., \& Ethofer, T. (2006). Cerebral processing of linguistic and emotional prosody: fMRI studies. Progress in Brain Research, 156, 249-268.

Wildgruber, D., Ethofer, T., Grandjean, D., \& Kreifelts, B. (2009). A cerebral network model of speech prosody comprehension. International Journal of Speech-Language Pathology, 11(4), 277-281.

Wilson, S. M., Galantucci, S., Tartaglia, M. C., Rising, K., Patterson, D. K., Henry, M. L., ... Gorno-Tempini, M. L. (2011). Syntactic processing depends on dorsal language tracts. Neuron, 72(2), 397-403.

Witteman, J., Van Heuven, V. J. P., \& Schiller, N. O. (2012). Hearing feelings: A quantitative meta-analysis on the neuroimaging literature of emotional prosody perception. Neuropsychologia, 50(12), 27522763.

Witteman, J., van ljzendoorn, M. H., van de Velde, D., van Heuven, V. J., \& Schiller, N. O. (2011). The nature of hemispheric specialization for linguistic and emotional prosodic perception: A metaanalysis of the lesion literature. Neuropsychologia, 49(13), 3722-3738.

Zarate, J. M. (2013). The neural control of singing. Frontiers in Human Neuroscience, 7, 237.

Zatorre, R. J., Belin, P., \& Penhune, V. B. (2002). Structure and function of auditory cortex: Music and speech. Trends in Cognitive Sciences, 6(1), 37-46.

\section{Supplementary Material}

Sound 1. Stimulus example of the prosody task with congruent prosody.

Sound 2. Stimulus example of the prosody task with incongruent prosody.

Sound 3. Stimulus example of the syntax task with S-O structure.

Sound 4. Stimulus example of the syntax task with O-S structure.

Sound 5. Example of a comprehension question in the syntax task. 


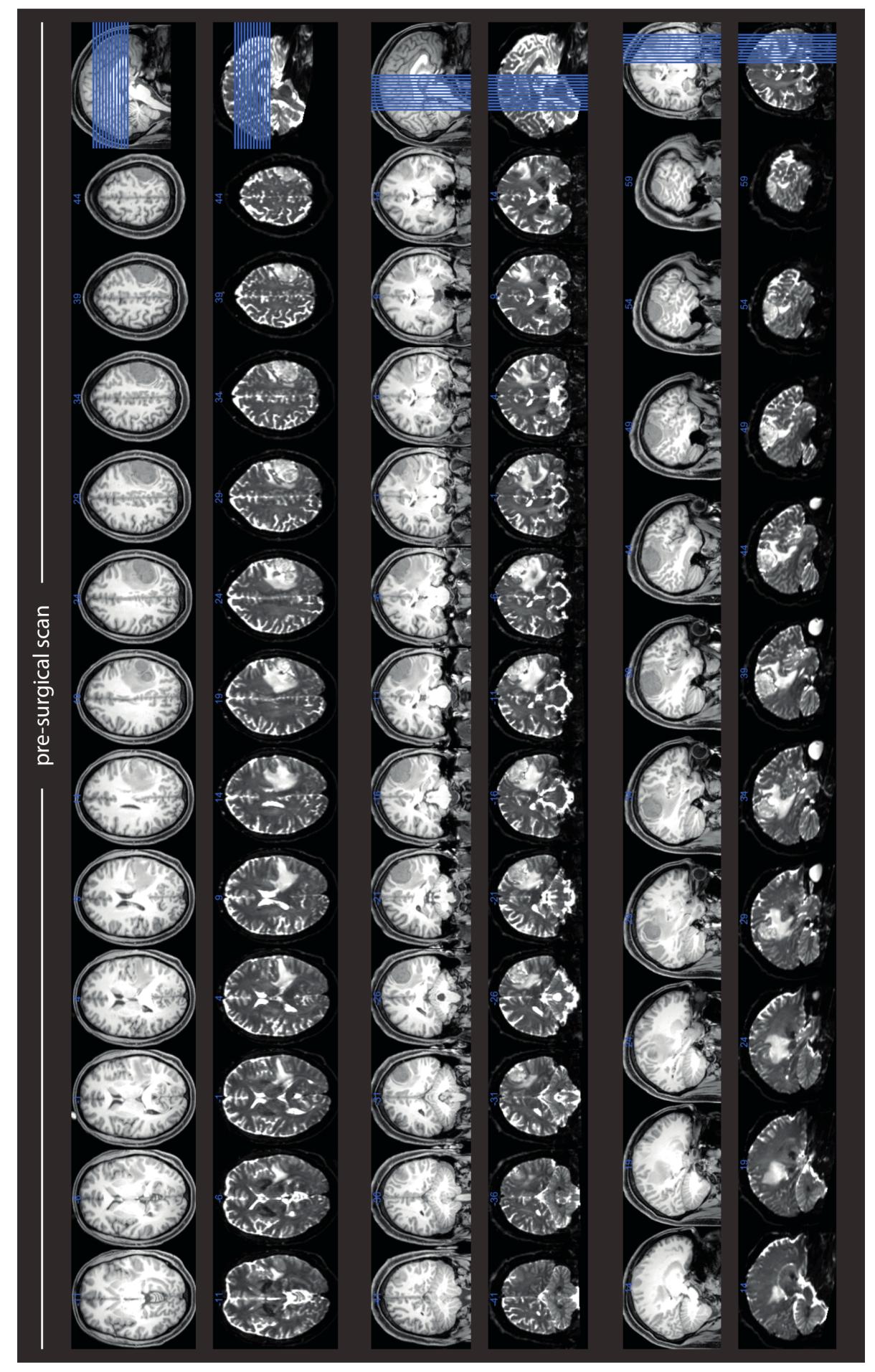

Supplementary Figure 1. Multi-slice view of pre-surgical scans. Depicted are axial, coronal and sagittal views of $\mathrm{T}_{1^{-}}$ weighted anatomical images for good visibility of the tumor (dark gray in upper lines) as well as the corresponding b0 images for good visibility of the edema (bright shadow in lower lines). 


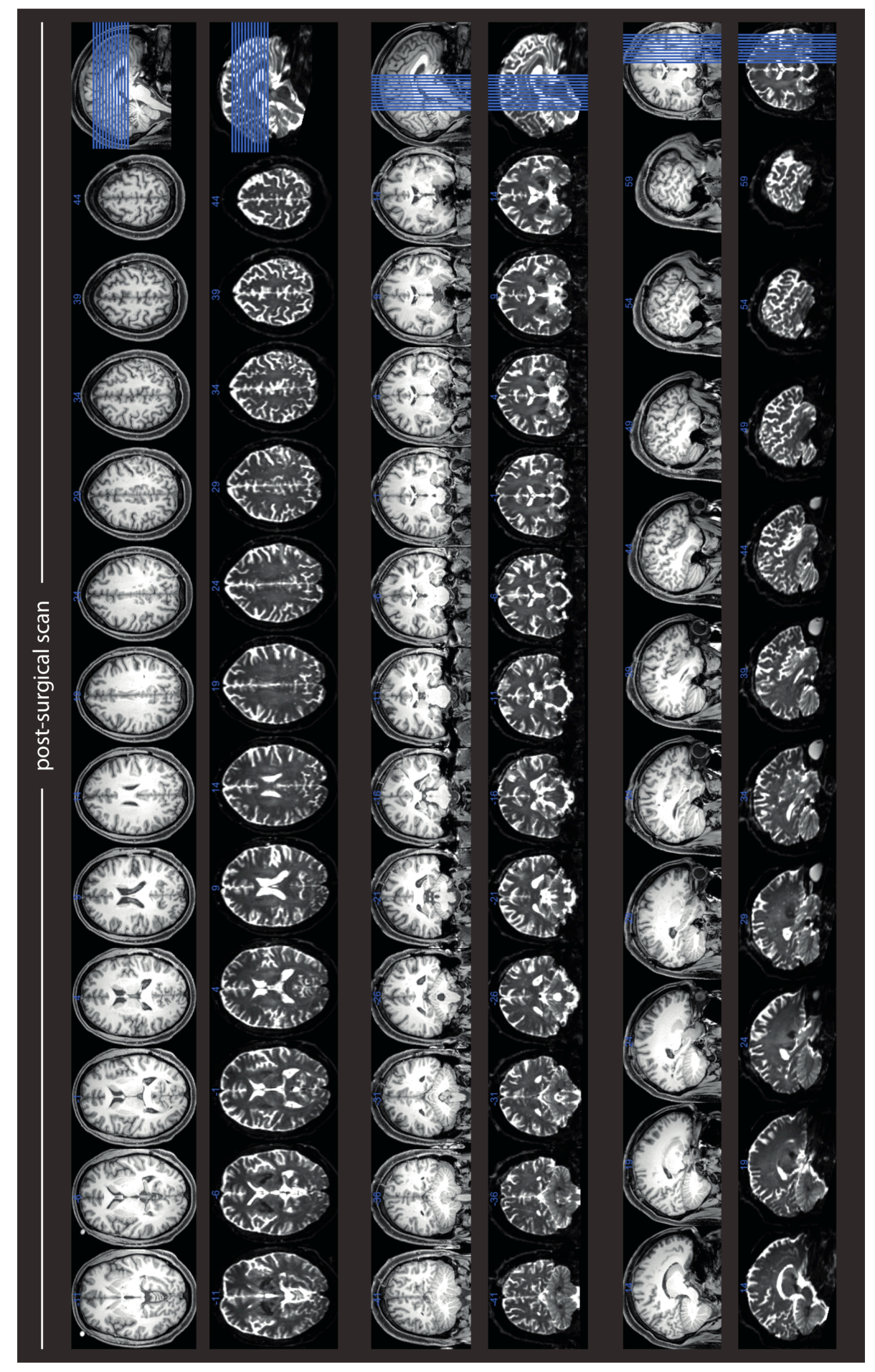

Supplementary Figure 2. Multi-slice view of post-surgical scans. Depicted are axial, coronal and sagittal views of $\mathrm{T}_{1^{-}}$ weighted (upper lines) and b0 images (lower lines) to illustrate the precision of tumor resection and complete edema reabsorption. 